TECHNOLOGY FEATURE

\title{
Sharpening the tools of RNA interference
}

If you want to silence a single gene with relative ease, look no further than RNA interference (RNAi). Fast becoming the knockdown method of choice in many systems, RNAi reagents such as commercial small inhibitory RNAs (siRNAs) and specialized media are now widely available to streamline your work. Caitlin Smith sizes up some of the new tools available for RNAi.

Billed as the breakthrough of 2002 by Science magazine, RNAi has been steadily gaining in repute, accessibility and utility. And no wonder - the ability to silence (or at least, downregulate) a gene in 24-48 hours with relative ease is an attractive thing. Still more tantalizing is the promise of RNAi in treating disease, by turning off the genes that cause malaise with minimal nonspecific effects.

The mechanism of RNAi was first discovered in plants and in worms, and soon after described in mammalian and other systems. Upon introduction of a doublestranded RNA (dsRNA) into a cell, the enzyme Dicer cleaves the dsRNA into fragments of 21-23 nucleotides each, called siRNAs. The siRNAs then associate with several proteins to form the RNAi-induced silencing complex (RISC), which directs the siRNAs to the target RNA sequence. Then the double-stranded siRNA unwinds, leaving the antisense strand to signal degradation of the mRNA sequence by endonucleases and exonucleases.

Although the introduction of longer, exogenous dsRNA molecules into cells triggers RNAi in plants and worms, in mammalian cells such molecules initiate a nonspecific type I interferon response leading to cell death. This response can be circumvented by using synthetic versions of the shorter siRNA molecules in mammalian systems. But this is not the only pitfall to befall RNAi researchers-designing the right siRNAs for your experiment can be a very tricky business.

\section{Challenges in designing siRNAs and available tools}

There are a myriad of design algorithms to help you design your siRNAs, some proprietary and some publicly available. Additionally, some companies offer predesigned siRNAs if you do not want to have a hand in the design. Most siRNA aficionados agree, though, that you should start with more than one siRNA. "Over the past few years, design algorithms have gotten better at predicting siRNAs that are effective at knocking down their targets," remarks Kathy Latham, marketing manager at Ambion. "But we still have a ways to go before we get to the point where experiments can be done with a single siRNA. Indeed, most journal article reviewers require that gene silencing results be confirmed with a second effective siRNA to a target before an article is accepted for publication."

The various design algorithms and online tools available are too numerous to list. One well-known design algorithm was introduced by researchers at Dharmacon. The process is known as rational siRNA design, and the algorithm is offered as a free online tool on Dharmacon's website, and is also available as an Excel template written by Maurice Ho, a researcher at the Hong Kong University of Science and Technology (http://boz094.ust.hk/RNAi/ siRNA). Rational siRNA design is based on a point system that was developed after researchers examined how efficiently 180 different siRNAs silenced the target mRNA of two genes. Invitrogen's design algorithm won the Frost and Sullivan Excellence in Research Award in 2004, and their BLOCK-iT RNAi Designer online tool allows you to select the design algorithm that you want to use: either their proprietary algorithm or one or more patterns based on the commonly used design method pioneered by Thomas Tuschl and

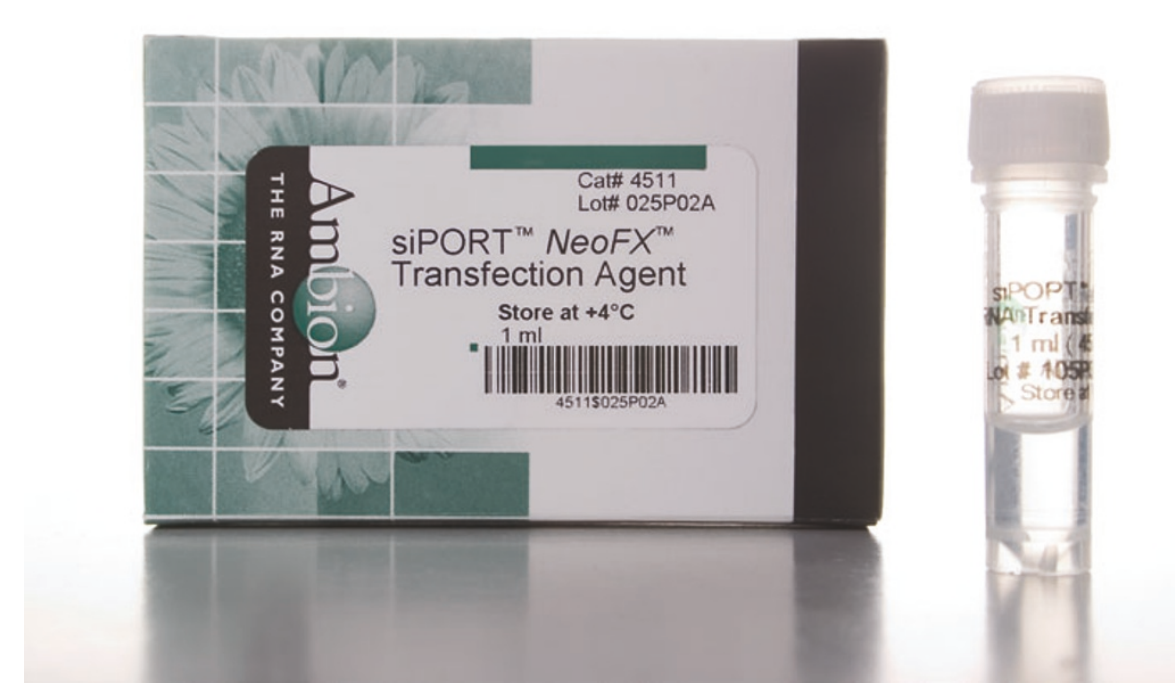

Ambion's siPORT NeoFX transfection reagent. (Courtesy of Ambion.) 


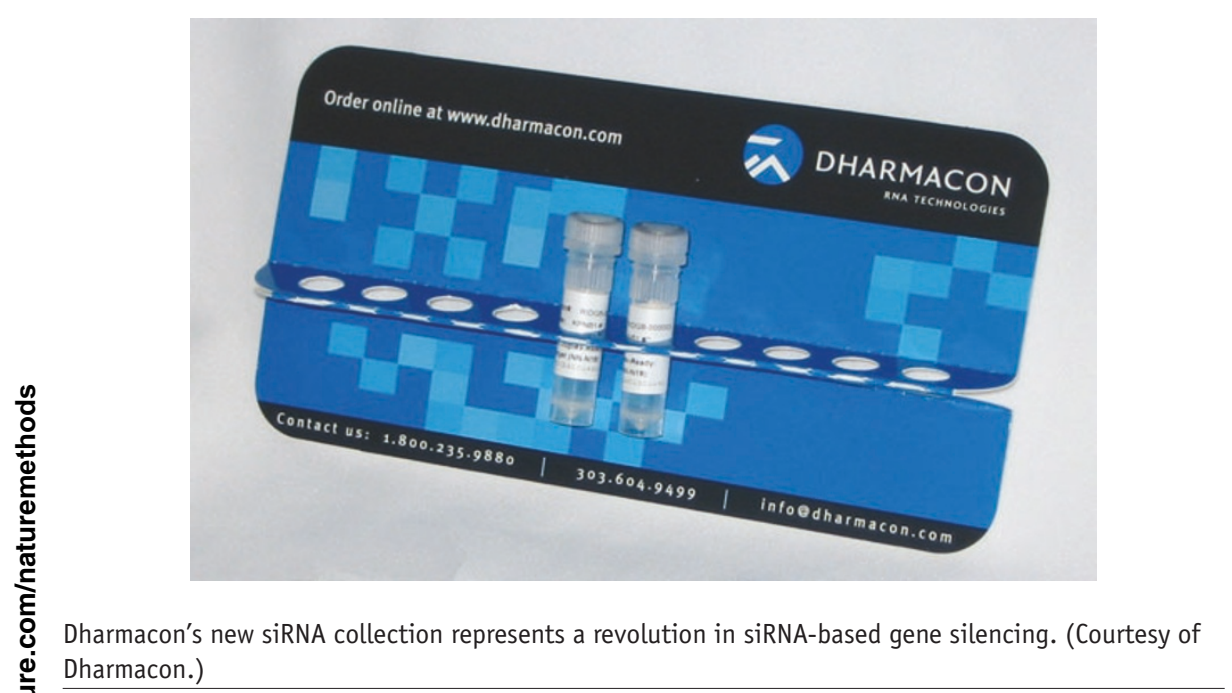

colleagues. Alternatively, a noncommercial online design tool is offered through the Whitehead Institute for Biomedical Research, Bioinformatics and Research Computing (http://jura.wi.mit.edu/ bioc/siRNAext/). This tool also provides options for design algorithms and is free after registration.

No matter which algorithm you use, however, there is no guarantee that the resulting siRNA will be effective enough for your experiment. Christophe Echeverri, the CEO and CSO of Cenix BioScience, explains that "the top algorithms yield likelihoods of about $80-90 \%$ that the resulting siRNAs will yield at least 70\% drops in target mRNA levels after 48 hours in well-optimized conditions in 'easy' human cells such as HeLa cells. This only gives users a statistical 'confidence factor', which is very useful, yes, but not a guarantee of performance."

Another problem in designing synthetic siRNAs is that not all targets are created equal, and there is no way to know the nature of your target before you begin. "Some targets are 'easy', says Mark Behlke, vice president of molecular genetics at Integrated DNA Technologies (IDT), "for which many or even most siRNAs seem to work when using a good design algorithm, whereas some targets are 'hard'" meaning that most siRNAs do not work well despite using a good design algorithm to create these siRNAs. "Fortunately, it appears that only about $10 \%$ of targets are hard," continues Behlke. "What makes a target hard? Current speculation revolves around the amount of 


\section{BOX 1 WHAT ARE PREVALIDATED SIRNAS?}

Strong opinions abound concerning the meaning of the words 'validated' or 'prevalidated' when it comes to siRNA molecules. But what exactly do these terms mean? Unfortunately for the RNAi novice who just wants to purchase the best reagents for the money, 'validated' can mean different things from different companies, and it is vital to understand companies' claims before you buy.

According to Mike Deines, vice president of sales and marketing at Dharmacon these terms may "create a false sense of security for the researcher." For example, he says that most validated siRNAs are synthesized "without taking offtarget effects into consideration. As a result, although these collections may provide strong knockdown of their intended target, they may also have significant off-target signatures." He also points out that validation studies are sometimes performed with exogenously expressed siRNAs. "Unfortunately," says Deines, "the efficacy of silencing endogenously expressed mRNA may not always correlate with exogenously expressed mRNA."

Behlke cautions that siRNAs 'validated' in one set of experimental conditions may not show as much knockdown in a different set of conditions. "It is important to keep in mind that although a reagent has been 'validated,' it still might not be sufficiently potent for experimental needs," he says. We recently helped one customer who was using a 'validated' duplex from another vendor against a human oncology target, which, while it did 'work', only reduced target mRNA levels by $70 \%$ when used at $100 \mathrm{nM}$ concentration. Although this reagent was 'validated,' it did not really knock down the target to levels needed by the investigator. A duplex from our TriFECTa kit against that target resulted in knockdown of $>90 \%$ at $1 \mathrm{nM}$ for that same target. This more potent reagent better served the experimental needs."

Echeverri points out another potential source of confusion for researchers - that design algorithms can be validated, while "the vast majority of the resulting siRNAs are indeed predesigned but not individually prevalidated." Instead, validation data sets give researchers a prediction of how the individual siRNAs designed by the algorithm are likely to behave under certain experimental conditions. "But of course," says Echeverri, "this offers no guarantee that every siRNA design will work perfectly in every other case, [and in] every other cell line." stable folding [for] secondary and tertiary structure, present in each individual mRNA. Another problem lies in the fact there is no universally applicable definition of what constitutes 'validated knockdown."'

\section{Commercial synthetic siRNAs}

With RNAi's recent explosion in popularity, more and more companies have been

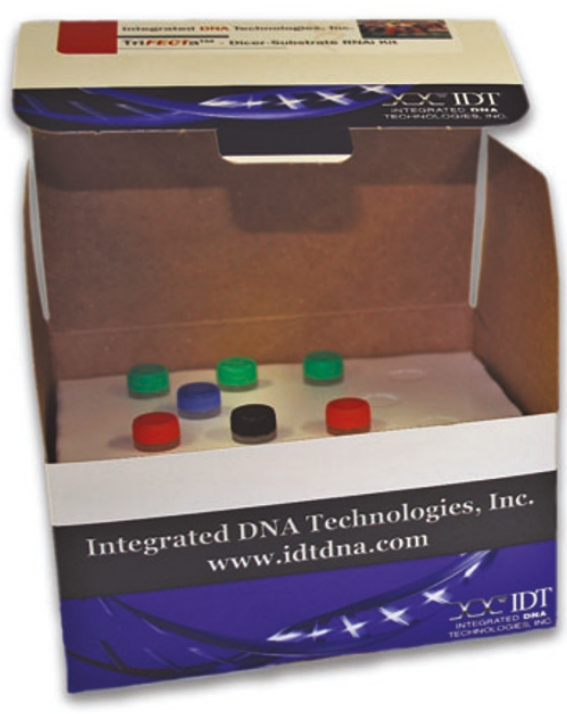

answering the demand for commercially available synthetic siRNAs. Many companies now allow you to choose from among custom oligonucleotides designed by you or the company as well as prevalidated, predesigned oligos (Box 1).

Ambion offers an extensive range of siRNA reagents, including both custom and predesigned varieties. Their Silencer

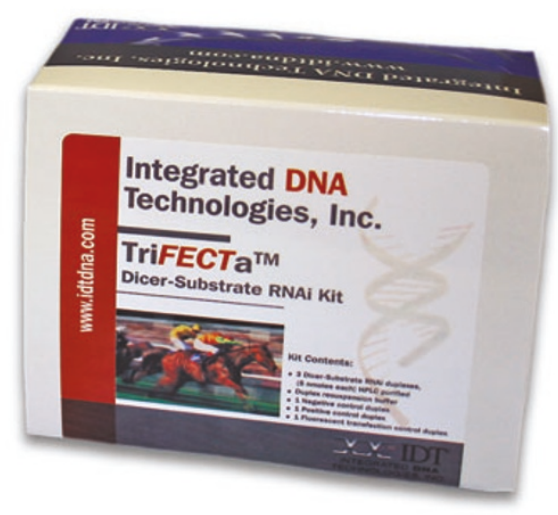

The TriFECTa Kit for siRNA transfection by Integrated DNA Technologies. (Courtesy of Integrated DNA Technologies.) line of predesigned siRNAs uses one of the most validated algorithms available. "The result is that when a customer obtains three siRNAs to a particular target, Ambion can guarantee that at least two of those will reduce target mRNA levels by $70 \%$ or more," says Latham. The design algorithm that is used to generate the Silencer line was developed by Cenix BioScience "and has been backed up by one of the industry's best, most in-depth experimental datasets, which helped set the standards for how siRNA libraries now get validated," says Echeverri. "This is a continually evolving process, so we are now hard at work on the next generation of siRNA designs."

Dharmacon's rational design algorithm underlies their SMARTpool reagent, which they claim offers a guaranteed $75 \%$ minimum target knockdown efficiency, with effectiveness reaching 95\%. The SMARTpool reagent is a mixture of four individual siRNAs, each aimed at a different region of the same target. Like Dharmacon, IDT also sells siRNAs as a mixture, although this 'pooling' of reagents is controversial (see Box 2). Behlke says, "We recommend testing a set of three [siRNA] duplexes and encourage this practice by bundling components into our TriFECTa kits, which include three target-specific duplexes, a positive 
control, a negative control and a dyelabeled transfection control in kit form."

Scientists at IDT have found it beneficial to use the natural processing pathway, linking Dicer cleavage with RISC loading in the cell. IDT offers 27-mer Dicersubstrate siRNAs, duplexes that enter the RNAi processing machinery one step before the traditional 21-mer siRNAs.
Invitrogen also offers a distinct twist with their Stealth line of siRNAs, which are chemically modified to have two unique features. "First, they have chemical modifications on the sense strand that inactivate that strand, leaving only the antisense strand to target the gene of interest," explains Peter Welch, director of gene expression profiling R\&D at Invitrogen.

\section{BOX 2 TO POOL, OR NOT TO POOL?}

No company will guarantee near-perfect knockdown with any single siRNA, but many offer high-percentage silencing with a mixture of three or four siRNAs. Such pooling is controversial, though; some find it efficient and cost-effective, but with a mixture one does not know which siRNA is active without painstakingly testing effective mixtures for individual siRNA characteristics.

Invitrogen's Welch believes that pooling is never a good idea. "While some researchers find value in pooling to save time during an initial large-scale screen, I do not think pooling siRNAs is a good idea in general-you have more work to follow up because you have to deconvolute whatever was in the pool. This includes verifying that each duplex in the pool is targeting the gene of interest, and knockdown of the target correlates with the phenotype without any off-target effects," he says. "A much better approach in the long run is to have two or three independent siRNAs targeting your gene of interest, all showing the same phenotype." Similarly, Latham, of Ambion, believes that "you can save yourself a step by bypassing the experiment with the siRNA pool, and going straight to the experiment with at least three individual siRNAs." Latham also comments that scientists at Ambion consistently find the rates of false positives and false negatives to be "significantly higher with pooled siRNAs than with three individual siRNAs per target."

Echeverri, from Cenix BioScience, sees some advantages to each method: "We've found that pools will typically miss about $15-20 \%$ of those hits that could have been detected with the individual siRNAs," even though the percentage varies depending on the assays used. "Of course, there is a major trade-off here: cost versus detection sensitivity," he continues. "For those who really need to minimize their rate of false negatives and can afford the use of multiple individual siRNAs, we'd recommend that pooling be avoided. If costs are a bigger limiting factor than the worry of missing some hits, then pooling may make sense."

The consideration of false positives and false negatives is even more important in larger-scale projects, especially in high-throughput work. Eric Lader, associate director of research and development at Qiagen, believes that for researchers studying one or two genes, the complications of pooling are not justified. "Large-scale screening with pools can save reagents and time in the primary screen. However, the main drawback to pooling in high-throughput is the loss of resolution in the experiment. When only a single biologically active compound targeting a gene is used versus four independent siRNAs, borderline positives, obviously false positives and false negatives are much harder to distinguish."

Conversely, Dharmacon's Deines believes that pooling is always a good idea. "By targeting multiple locations on the target mRNA, pools minimize false negatives caused by sequence mutations, undocumented [single-nucleotide polymorphisms] and splice variants. In addition, pools of highly functional siRNAs tend to most closely resemble the silencing ability of the most functional siRNA in the pool, thus enhancing the overall chances of achieving potent gene knockdown. Pools have also been shown to generate fewer off-target effects. This effect is likely due in part to the concentration dependence of off-target effects, and in part due to elimination of toxicity observed with individual siRNAs. The other advantage of siRNA pools is economy. Using siRNA pools allows the researcher to achieve a high level of experimental confidence with fewer experiments." 
"The sense strand can enter RISC and knockdown genes, so inactivating this strand immediately removes any off-target potential of the sense strand. Second, we have employed a rigorous specificity algorithm, called Smith-Waterman, to help eliminate off-target effects" (see Box 3 ). The resulting product is Invitrogen's line of predesigned siRNAs called Stealth
Select, which are available for nearly all genes in rats, mice and humans as three nonoverlapping siRNAs per gene.

Dharmacon is also tackling the problem of off-target effects with the recent introduction of their new ON-TARGETplus siRNAs. According to Mike Deines, the new siRNA collection can give a "reduction in off-target effects of up to $90 \%$," allowing researchers to "spend far less time chasing false positive results and confusing phenotypes."

\section{Getting the message through the medium}

New varieties of media are being developed to facilitate the transfection of cells with synthetic oligos for RNAi. Among these are the common lipid-based and amine-based transfection reagents, which both offer high reproducibility with minimal toxicity. Different cell types have different requirements, however, and some trial and error is often required when using an unfamiliar cell type. Echeverri says: "There is no single magic bullet that works perfectly for all. With each new cell line, it is definitely worthwhile to test a panel (the wider the better) of different reagents, to find the optimum."

Scientists at IDT use a variety of cationic lipid reagents along with dye labels to optimize transfection conditions for each cell line. "We begin optimization using a dyelabeled RNA duplex, as this offers a visual assay, which is easy, inexpensive and rapid to perform," explains Behlke. "Having identified one or more lipids or protocols that appear to work, we test a positive control duplex (already known to work in other cell lines from the same organism) and assess the actual percentage knockdown (usually of a housekeeping gene). We then move on to [test] new target-specific duplexes."

Ambion's new siPORT NeoFX Transfection Agent, according to Latham, is especially effective for immortalized adherent cell types, such as HeLa cells. Ambion claims that it allows transfection to occur within one day rather than two, which can save time when optimizing protocols, for example. The new siPORT NeoFX was also designed for transfecting with lower siRNA concentrations (1-20 $\mathrm{nM}$ ), which may reduce the incidence of off-target effects.

Also optimized for use at lower siRNA concentrations is Invitrogen's new Lipofectamine RNAiMAX Transfection Reagent, specifically designed for RNAi and based on their popular Lipofectamine 2000 reagent. Invitrogen claims that this reagent offers optimal knockdown at $10 \mathrm{nM}$ siRNA—or even at $1 \mathrm{nM}$ when their Stealth siRNAs are being used. Another attraction of this product is its low cytotoxicity across a tenfold concentration range, which facilitates optimization. 


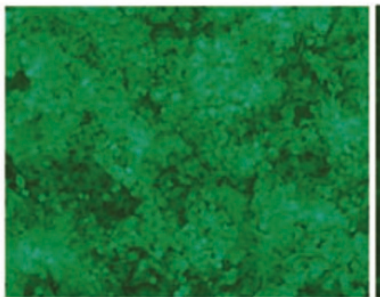

Control

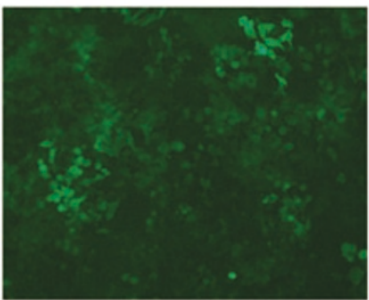

SilenceMag $4 \mathrm{nM}$ siRNA

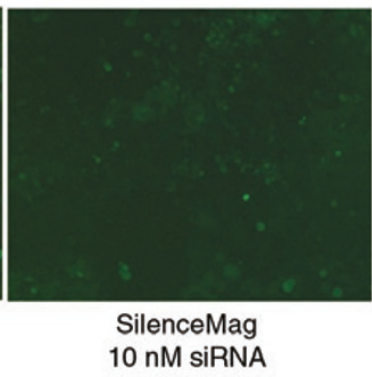

$10 \mathrm{nM}$ siRNA

HeLa cells stably expressing GFP, treated with OZ Biosciences' SilenceMag, and 4 or $10 \mathrm{nM}$ of siRNA targeting GFP. GFP expression $72 \mathrm{~h}$ after transfection was assayed by fluorescence microscopy. (Courtesy of $\mathrm{OZ}$ Biosciences.)

OZ Biosciences offers several reagents for siRNA work based on their magnetofection technology. OZ Biosciences' cell biology manager, Laurent Meunier, explains: "magnetic nanoparticles coated with proprietary molecules allow high delivery of small amount of siRNA (10 $\mathrm{nM})$ in common cell lines as well as in primary cells by applying a suitable magnetic field to concentrate siRNA onto the cell surface and promote cellular delivery. Our lipid-based transfection reagent, DreamFect, is often used for cotransfection and siRNA delivery with high efficiencies." Another reagent, SilenceMag, works similarly through application of a magnetic field and offers "very efficient delivery of siRNA, even with difficultto-transfect cells," according to Meunier. Additionally, OZ Biosciences is one of the few companies currently offering media designed for transfecting insect cells with siRNA. Their FlyFectin product is billed as a nontoxic, efficient reagent that yields reproducible results, and can be used with or without serum in culture and in highthroughput experiments.

\section{RNAi in vivo}

The therapeutic use of RNAi in vivo is not yet routine, but researchers are steadily progressing toward this goal. "The in vivo application of RNAi remains a key goal of the field, with huge potential for yielding important new insights," says Echeverri. "We are routinely using RNAi to conduct high-throughput genome-scale screens to identify new therapeutic drug targets in areas such as oncology, cardiovascular diseases, metabolic diseases as well as infectious diseases including malaria, and the list keeps growing. The same techniques are being used to validate these targets, that is, characterize their loss-offunction phenotypes as carefully as possible, as a way of predicting the effects of eventual therapeutic agents targeting those genes."

Most in vivo RNAi research falls under one of two headings: local or systemic applications. Local applications to several tissue types in vivo have already been demonstrated by several groups. Systemic application of RNAi has proven more challenging. Says Echeverrri, "several groups are trying to either come up with modified synthetic molecules delivered intravenously, or via various 'gene therapy' approaches (vector-driven expression) or transgenic approaches. If successful, these studies will allow a much more refined analysis of loss-of-function phenotypes in vivo, with the promise of delivering different levels of silencing triggered at different times during development." Such results may replace inducible knockouts in model systems.

Only recently have results emerged to prove the predicted therapeutic value of RNAi in systemic administration. Scientists at Alnylam Pharmaceuticals and Protiva Biotherapeutics recently announced the use of systemically delivered RNAi to silence the gene for apolipoprotein $\mathrm{B}$ (apoB), a protein involved in cholesterol metabolism. This is a major advance because the treatment resulted in a clinically significant reduction in cholesterol and low-density lipoproteins; in addition, the therapeutic dosage was appropriate for future human clinical studies.

In one study, ApoB was silenced using siRNA encapsulated in a liposomal formulation to facilitate delivery. In another 

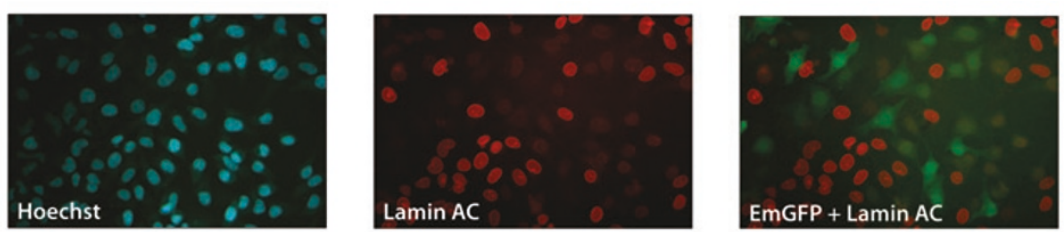

Demonstration of the Invitrogen BLOCK-iT(TM) Pol II RNAi system. Cells were transfected at 50\% efficiency with a vector in which EmGFP is coexpressed with a microRNA targeting lamin, using

Lipofectamine 2000 transfection reagent. After 48 h, cells expressing GFP (green) exhibit reduced levels of lamin (red) present, and vice versa, as indicated by immunostaining (right and middle). The left panel indicates cell labeling using the Hoechst nuclear stain. (Courtesy of Invitrogen.)

strategy used by Alnylam, therapeutic siRNAs were conjugated to a small molecule that promotes uptake into cells. "An example of such a modification is our work in which we coupled cholesterol to an siRNA targeting a gene expressed in hepatocytes and enterocytes of the small intestine," says Nagesh Mahanthappa, senior director of business development and strategy at Alnylam.

Despite the recent success stories, there is a ways to go before RNAi therapeutics become commonplace. "The issue of demonstrating targeting specificity for in vivo RNAi is not a trivial one," cautions Echeverri, "and will have to be convincingly addressed before the method can deliver its full potential." Multiple factors must be taken into account when developing siRNA molecules as potential drug candidates. According to Mahanthappa, "these include specificity for the target of interest, lack of induction of an interferon response, amenability to chemical modification to promote in vivo stability .... and [the] ability to scale production to the gram or multi-gram scale for clinical use." Nevertheless, considerable excitement remains for the promise of this technology. As Welch comments, "once the delivery issues are solved, I wholeheartedly believe that RNAi will be successful at the whole-organism level."

Most people agree that the key to the success of therapeutic in vivo RNAi lies in improving delivery. Behlke comments that while chemically modified siRNAs that have nuclease resistance and improved properties for in vivo use (such as escape from activation of the innate immune system) are easily made, "their delivery remains a major problem. The availability of good in vivo delivery tools for use by general researchers would have a great impact on accelerating progress in this field."

Several companies are now selling siRNAs and reagents aimed at researchers doing in vivo work. For example, Ambion offers their Silencer In Vivo Ready siRNAs, which are prepared with extra

\section{BOX 3 THE QUANDARY OF OFF-TARGET EFFECTS}

The problem of off-target effects - the unintentional silencing of nontargeted genes - continues to plague RNAi researchers. The problem of off-target effects is alarming because "siRNAs designed to target one gene could suppress the expression of dozens of unrelated genes," observes Deines. "While the levels of gene downregulation by this mechanism are modest (two- to fivefold), studies by several labs have now shown that offtarget effects can generate measurable phenotypes."

Yet off-target effects - and how to avoid them-are still incompletely understood. In initial RNAi research, emphasis was placed on maximizing silencing efficacy. "It is now clear that, like essentially any other types of biologically active molecule, literally all reagents designed to trigger RNAi, whether short or long dsRNA, whether made of 'naked RNA' or including chemical modifications, carry non-negligible risks of generating [off-target effects]," explains Echeverri. The emphasis then shifted to minimizing off-target effects, with further unintended effects. "Precautions taken to reduce [off-target effects] when designing siRNAs often led the designer to leave out candidate siRNA sequences that may have represented excellent silencers. Some of these precautions now emerge as rather heavy-handed, erring on the side of caution, and perhaps too often sacrificing potentially good silencers for unjustified fears of [off-target effects]," says Echeverri. Today, as a better balance between silencing efficacy and off-target effects is achieved, "it is clear that reagent design alone cannot rule out the [offtarget effect] risks, so specificity can only be ascertained through rigorous experimental controls."

Invitrogen uses the Smith-Waterman algorithm to minimize off-target effects in the development of their predesigned Stealth Select line of siRNAs. According to Welch, "SmithWaterman analysis, when used on the entire target mRNA [( $5^{\prime}$ untranslated region (UTR) plus open reading frame plus 3' UTR)], will detect and eliminate siRNAs whose 'seed region' is complementary to a potential off-target gene." A recent article from scientists at Dharmacon and Agilent Technologies showed that Smith-Waterman and other algorithms were ineffective in minimizing off-target effects, except for when the overall identity of the off-target gene was nearly perfectly matched to the target gene ${ }^{1}$. Rather, they concluded that most off-target effects occur because of perfect matches between the 3' UTR in the off-targeted gene, and the seed region (nucleotides $2-7$ or 2-8) of the siRNA antisense strand.

What more can you do to avoid off-target effects? The answer is not entirely clear yet, but may depend in part on your experimental system-the mechanisms involved in generating off-target effects may differ in different organisms. Stay tuned for the end of the off-target effect story, as many groups are now working to gain a greater understanding of off-target effects and how they work. 
purification and testing, ready for animal injection. These siRNAs can be synthesized from custom sequences or from Ambion's Silencer Pre-designed or Validated line. Mirus Bio offers the TransIT-QR Delivery Solution that can be used to deliver naked siRNA to the mouse liver via hydrodynamic tail vein injections. Polyplus Transfection offers their jet-PEI transfection reagent for local or systemic delivery of siRNA in vivo, claiming to have proven success in studies with intratumoral delivery and intravenous delivery.

Though it is a relatively new technique, it is clear that RNAi is no passing fad, and is here to stay. "In the future, RNAi will be used by virtually everyone wanting to study gene function, whether they are using cultured-cell or whole-organism models," says Latham. "In addition, more and more emphasis is likely to be placed on the therapeutic potential of siRNAs for treating human disease."

\section{Caitlin Smith is a science writer in Portland, Oregon (caitlin.smith@comcast.net).}

1. Birmingham, A. et al. 3' UTR seed matches, but not overall identity, are associated with RNAi off-targets. Nat. Methods 3, 199-204 (2006). 
TECHNOLOGY FEATURE

\section{SUPPLIERS GUIDE: COMPANIES OFFERING REAGENTS AND TOOLS FOR RNAi WORK}

\section{Company}

Alnylam

Amaxa Biosystems

Ambion

BioCat

BioChain

Boca Scientific

o Cenix Bioscience

Clontech

Dharmacon

Eurogentec

Gene Link

Geneservice

Genlantis

GenScript

Imgenex

Integrated DNA Technologies

Invitrogen

InvivoGen

Mirus

Molecula

MWG Biotech

New England BioLabs

Novagen

OligoEngine

Open Biosystems

OrbiGen

OZ Biosciences

Panoramics

Polyplus Transfection

Promega

Protiva

Qiagen

RNAx

Roche Applied Science

Sigma Aldrich

Sirna Therapeutics

Stratagene

SuperArray Bioscience Corporation

Tebu-Bio

Upstate

\section{Web address}

http://www.alnylam.com

http://www.amaxa.com

http://www.ambion.com

http://www.biocat.de

http://www.biochain.com

http://www.bocascientific.com

http://www.cenix-bioscience.com

http://www.clontech.com

http://www.dharmacon.com

http://www.eurogentec.com

http://www.genelink.com

http://www.geneservice.co.uk

http://www.genlantis.com

http://www.genscript.com

http://www.imgenex.com

http://www.idtdna.com

http://www.invitrogen.com

http://www.invivogen.com

http://www.mirus.com

http://www.molecula.com

http://www.mwg-biotech.com

http://www.neb.com

http://www.emdbiosciences.com

http://www.oligoengine.com

http://www.openbiosystems.com

http://www.orbigen.com

http://www.ozbiosciences.com

http://www.panoramics.com

http://www.polyplus-transfection.com

http://www.promega.com

http://www.protivabio.com

http://www.qiagen.com

http://www.rnax.de

http://www.roche-applied-science.com

http://www.sigmaaldrich.com

http://www.sirna.com

http://www.stratagene.com

http://www.superarray.com

http://www.tebu-bio.com

http://www.upstate.com 